\title{
Ordered Pair Semantics and Negation in LP
}

\author{
Matthew Clemens \\ Department of Philosophy, \\ Siena College \\ Loudonville, NY 12211 \\ matt.eby.clemens@gmail.com
}

\begin{abstract}
This paper presents a novel semantic framework for the many-valued paraconsistent logic LP. By using this framework, we can preserve a significant classical intuition about negation, namely that the negation operator reverses truth-value.
\end{abstract}

\section{Negation and truth-values}

Elementary presentations of negation in classical logic often utilize the intuitive idea that the negation operator reverses truth-value, ${ }^{1} \neg \alpha$ is true when $\alpha$ is false; and $\neg \alpha$ is false when $\alpha$ is true. By contrast, standard presentations of negation in LP suggest a division in the cases at issue; ${ }^{2}$ classical truth-values are reversed by the negation operator, but truth-value is static, i.e., preserved by the negation operator, in the case of the 'middle' (both true and false) truth-value. Where $i$ denotes the semantic value both true and false, and the set of designated values is $\{1, i\}$, the standard truth table for negation in LP can be given as follows:

\begin{tabular}{l|l}
$\neg$ & \\
\hline 1 & 0 \\
$i$ & $i$ \\
0 & 1
\end{tabular}

This note provides a formal framework for the semantics of the connectives of LP that fully preserves the intuitive notion that negation reverses the truth-value of its negatum. As a result, a more uniform explanation of the behavior of negation in LP is available.

A natural thought about many-valued paraconsistent logics like LP is that some sentences of the language have more than one truth-value. In an early presentation of the logic that would come to be known as LP, Asenjo is explicit about this:

\footnotetext{
${ }^{1}$ See, e.g., N.J.J. Smith 2012 p. 127 and Tomassi 1999 p. 74 and p. 78. See also P. Smith 2003 p. 75 and p. 101 (using the term 'flip') and G. Hardegree Symbolic Logic: A First Course online (using the term 'opposite').

' 'LP' is the now standard name for the paraconsistent logic developed as early as 1966 (Asenjo 1966), and independently in 1979 (Priest 1979). See Beall and van Fraassen 2003, Beall 2010, Beall and Logan 2017, Priest 2008, or Restall 2005 for introductory expositions. For an early overview and historical presentation of paraconsistent logic, see Arruda 1980.
} 
"Let us assume that atomic propositions have either one or two truth values. Propositions (atomic or compound) will then either be true, false, or true and false. ... The truth value or values of $A \star B$ (where $\star$ stands for any binary connective) are the value or values that result from giving $A$ and $B$ all possible combinations of the truth values." 3

In this way, so-called glutty sentences (or 'antinomies', as Asenjo called them) can be thought of as having two truth-values. Indeed Asenjo goes on to distinguish antinomic sentences from "single-valued" propositions. ${ }^{4}$

There is also a natural sense in which every sentence of the language of LP has a two-part semantic character. Here is Priest introducing the semantic values of LP:

"In the context of LP, the value $i$ is thought of as both true and false.

Consequently, 1 and 0 have to be thought of as true and true only, and false and false only, respectively." ${ }^{\prime 5}$

A formal framework that makes the two-part semantic character of every sentence in LP explicit has the benefit of allowing for the standard understanding of negation as the connective that reverses truth-value.

\section{Ordered pair semantics}

In order to capture this two-part semantic character, we can utilize ordered pairs, such that a sentence with the semantic value true and true only gets the value $\langle 1,1\rangle$, a sentence with the semantic value false and false only gets the value $\langle 0,0\rangle$, and a sentence with the semantic value both true and false gets the value $\langle 1,0\rangle$, or the semantic value $\langle 0,1\rangle$. Given this set of semantic values, $\mathcal{V}=\{\langle 1,1\rangle,\langle 1,0\rangle,\langle 0,1\rangle,\langle 0,0\rangle\}$, we define the set of designated values, $\mathcal{D}=\{\langle 1,1\rangle,\langle 1,0\rangle,\langle 0,1\rangle\}$. We also adopt the usual understanding of validity in the many-valued paraconsistent context, i.e., any interpretation in which the premises of an argument are all designated is an interpretation where the conclusion is also designated.

Using an ordered pair semantics, where $S$ is the set of all sentences in LP, and $v$ a function from $S$ into the set of semantic values $\mathcal{V}$, we define negation as follows. ${ }^{6}$

$$
\begin{array}{llll}
v(\neg \alpha)=\langle 1,1\rangle & \text { iff } & v(\alpha)=\langle 0,0\rangle \\
v(\neg \alpha)=\langle 1,0\rangle & \text { iff } & v(\alpha)=\langle 0,1\rangle \\
v(\neg \alpha)=\langle 0,1\rangle & \text { iff } & v(\alpha)=\langle 1,0\rangle \\
v(\neg \alpha)=\langle 0,0\rangle & \text { iff } & v(\alpha)=\langle 1,1\rangle
\end{array}
$$

The truth table for negation can be then given as follows.

\footnotetext{
${ }^{3}$ Asenjo 1966 p. 103.

${ }^{4}$ Ibid.

${ }^{5}$ Priest 2008 p. 124.

${ }^{6}$ Alternatively, by defining subtraction on ordered pairs as $\langle n, m\rangle-\langle x, y\rangle=\langle n-x, m-y\rangle$, we can also define negation as $v(\neg \alpha)=\langle 1,1\rangle-v(\alpha)$.
} 


\begin{tabular}{c|c}
$\neg$ & \\
\hline$\langle 1,1\rangle$ & $\langle 0,0\rangle$ \\
$\langle 1,0\rangle$ & $\langle 0,1\rangle$ \\
$\langle 0,1\rangle$ & $\langle 1,0\rangle$ \\
$\langle 0,0\rangle$ & $\langle 1,1\rangle$
\end{tabular}

To define disjunction and conjunction, we utilize the following ordering on semantic values.

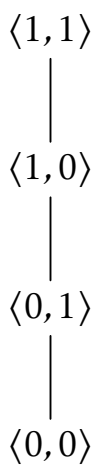

Using this ordering, we can adopt standard definitions of disjunction and conjunction in LP. ${ }^{7}$

$$
\begin{aligned}
& v(\alpha \vee \beta)=\max \{v(\alpha), v(\beta)\} \\
& v(\alpha \wedge \beta)=\min \{v(\alpha), v(\beta)\}
\end{aligned}
$$

These definitions then yield the following truth tables for disjunction and conjunction.

\begin{tabular}{c|cccc}
$\vee$ & $\langle 1,1\rangle$ & $\langle 1,0\rangle$ & $\langle 0,1\rangle$ & $\langle 0,0\rangle$ \\
\hline$\langle 1,1\rangle$ & $\langle 1,1\rangle$ & $\langle 1,1\rangle$ & $\langle 1,1\rangle$ & $\langle 1,1\rangle$ \\
$\langle 1,0\rangle$ & $\langle 1,1\rangle$ & $\langle 1,0\rangle$ & $\langle 1,0\rangle$ & $\langle 1,0\rangle$ \\
$\langle 0,1\rangle$ & $\langle 1,1\rangle$ & $\langle 1,0\rangle$ & $\langle 0,1\rangle$ & $\langle 0,1\rangle$ \\
$\langle 0,0\rangle$ & $\langle 1,1\rangle$ & $\langle 1,0\rangle$ & $\langle 0,1\rangle$ & $\langle 0,0\rangle$ \\
$\wedge$ & $\langle 1,1\rangle$ & $\langle 1,0\rangle$ & $\langle 0,1\rangle$ & $\langle 0,0\rangle$ \\
\hline$\langle 1,1\rangle$ & $\langle 1,1\rangle$ & $\langle 1,0\rangle$ & $\langle 0,1\rangle$ & $\langle 0,0\rangle$ \\
$\langle 1,0\rangle$ & $\langle 1,0\rangle$ & $\langle 1,0\rangle$ & $\langle 0,1\rangle$ & $\langle 0,0\rangle$ \\
$\langle 0,1\rangle$ & $\langle 0,1\rangle$ & $\langle 0,1\rangle$ & $\langle 0,1\rangle$ & $\langle 0,0\rangle$ \\
$\langle 0,0\rangle$ & $\langle 0,0\rangle$ & $\langle 0,0\rangle$ & $\langle 0,0\rangle$ & $\langle 0,0\rangle$
\end{tabular}

It might be suggested that this way of presenting the logic adds a 'fourth' truthvalue (both false and true) to the standard semantic framework for LP. However, as

${ }^{7}$ See, e.g., Beall 2009, Beall 2011.

Australasian Journal of Logic (17:5) 2020, Article no. 1 
there is no sense in which the standard 'third' truth-value of LP has any privileged ordering of true and false, the ordered pair framework does not make a substantive alteration to the semantics. That is, there is no sense in which, on a standard LP account, the Liar sentence, for example, is true and false in LP, but not false and true in LP. ${ }^{8}$

\section{A natural interpretation}

The ordered pair semantic framework allows for a natural and uniform explanation of the behavior of negation in LP, which is exactly parallel to the kind of general explanation often given for negation in classical logic. Rather than explaining the behavior of negation in LP with respect to sentences assigned classical truthvalues and then separately explaining the behavior of negation in LP with respect to glutty/antinomic sentences, we can simply point out that the negation operator in LP reverses the truth value of the negatum. ${ }^{9}$

Of course, we still have a natural way of understanding the fact that a glutty/ antinomic sentence remains glutty/antinomic when negated; the negation operator has reversed all the truth-value assignments for the original sentence, so that the negation of a sentence that is both true and false is both false and true, and vice versa. In the case of a glut/antinomy, the truth-value reversing nature of the negation operator leaves us with a glut/antinomy.

Finally, as mentioned above, the ordered pair semantic framework offers a clear formal representation of the idea that every sentence in LP has a two-part semantic character. For example, a sentence assigned the semantic value $\langle 1,1\rangle$ can be naturally understood as capturing the idea that such a sentence is 'true and true only' in LP. $^{10}$

\footnotetext{
${ }^{8}$ The ordered pair semantics of this paper shares important similarities with the four-valued semantics of FDE originating in work of Dunn 1976 and Belnap 1977, and indeed also with the generalization of Belnap-Dunn semantics found in, e.g., Wansing 1993 and Pynko 1995, and with the non-deterministic semantics developed in work of Avron (see Avron and Zamansky 2011 for an overview). However, the point made above, i.e., that the ordered pair semantics sketched here does not add a semantically distinct fourth value to LP, is useful in thinking about the dissimilarities. While the ordered pair semantics presented in this paper includes two 'middle' semantic values $(\langle 1,0\rangle$ and $\langle 0,1\rangle)$, these middle values are disanalogous to the two 'middle' values of FDE-based systems, which contrast in a semantic sense with one another; in FDE-based systems, one middle value expresses the overdetermination of truth-value ('both true and false') and one middle value expresses the absence of truth-value ('neither true nor false'). Another way to highlight the difference at issue here is to note that while FDE-based logics are both paraconsistent and paracomplete, the ordered pair semantics presented in this paper does not change the fact that LP is solely paraconsistent.

${ }^{9}$ The overarching similarity between negation in the many-valued paraconsistent context and negation in the classical context is highlighted by Beall and Logan in their 2017. In presentating the connectives of FDE, they suggest that the relevant semantics are "fairly classical", making reference to negation in particular:

"For example, negations are true just if their negatums are false (even if the negatum also happens to be true), and are false just if the negatum is true (even if the negatum also happens to be false) - just as in the classical picture." (Beall and Logan 2017 p. 195)

${ }^{10}$ I wish to thank Colin Caret and Edwin Mares for helpful comments on earlier versions of this paper.
}

Australasian Journal of Logic (17:5) 2020, Article no. 1 


\section{References}

[1] Arruda, A.I. 1980. "A Survey of Paraconsistent Logic", in A.I. Arruda, R. Chuaqui, and N.C.A. da Costa, eds. Mathematical Logic in Latin America (pp. 1-41). Amsterdam: North-Holland.

[2] Avron, A. and Zamansky, A. 2011. "Non-Deterministic Semantics for Logical Systems", in D. Gabbay and F. Guenthner (eds.), Handbook of Philosophical Logic, Volume 16, pp. 227-304. Springer.

[3] Asenjo, F.G. 1966. "A Calculus of Antinomies", Notre Dame Journal of Formal Logic 7: 103-105.

[4] Beall, J. 2009. Spandrels of Truth. Oxford: Oxford University Press.

[5] Beall, J. 2010. Logic: The Basics. New York: Routledge.

[6] Beall, J. 2011. "Multiple-Conclusion LP and Default Classicality", The Review of Symbolic Logic 4: 326-336.

[7] Beall, J. and Logan, S. 2017. Logic: The Basics. 2nd edition. New York: Routledge.

[8] Beall, J. and van Fraassen, B. 2003. Possibilities and Paradox: An Introduction to Modal and Many-Valued Logic. Oxford: Oxford University Press.

[9] Belnap, N. 1977. "A Useful Four-Valued Logic", in J.M. Dunn, and G. Epstein, (eds.), Modern Uses of Multiple-Valued Logic (pp. 8-37). D. Reidel Publishing Co.

[10] Dunn, J. 1976. "Intuitive semantics for first degree entailment and coupled trees", Philosophical Studies 29: 149-168.

[11] Hardegree, G. Symbolic Logic: A First Course. Available online: http://courses.umass.edu/phil110-gmh/MAIN/IHome-5.htm

[12] Priest, G. 1979. "Logic of Paradox", Journal of Philosophical Logic 8: 219-241.

[13] Priest, G. 2008. An Introduction to Non-Classical Logic: From If to Is. Cambridge: Cambridge University Press.

[14] Pynko, A.P. 1995. “Characterizing Belnap's logic via De Morgan’s laws”, Mathematical Logic Quarterly 41: 442-454.

[15] Restall, G. 2005. Logic: An Introduction. New York: Routledge.

[16] Smith, N.J.J. 2012. Logic: The Laws of Truth. Princeton: Princeton University Press.

[17] Smith, P. 2003. An Introduction to Formal Logic. Cambridge: Cambridge University Press.

[18] Tomassi, P. 1999. Logic. New York: Routledge.

[19] Wansing, H. 1993. The Logic of Information Structures. Berlin: Springer. 